\title{
Fractional baud-length coding
}

\author{
J. Vierinen \\ Sodankylä Geophysical Observatory, Sodankylä, Finland
}

Received: 24 March 2010 - Revised: 8 February 2011 - Accepted: 6 June 2011 - Published: 30 June 2011

\begin{abstract}
We present a novel approach for modulating radar transmissions in order to improve target range and Doppler estimation accuracy. This is achieved by using non-uniform baud lengths. With this method it is possible to increase subbaud range-resolution of phase coded radar measurements while maintaining a narrow transmission bandwidth. We first derive target backscatter amplitude estimation error covariance matrix for arbitrary targets when estimating backscatter in amplitude domain. We define target optimality and discuss different search strategies that can be used to find well performing transmission envelopes. We give several simulated examples of the method showing that fractional baudlength coding results in smaller estimation errors than conventional uniform baud length transmission codes when estimating the target backscatter amplitude at sub-baud range resolution. We also demonstrate the method in practice by analyzing the range resolved power of a low-altitude meteor trail echo that was measured using a fractional baud-length experiment with the EISCAT UHF system.
\end{abstract}

Keywords. Radio science (Ionospheric physics; Signal processing; Instruments and techniques)

\section{Introduction}

We have previously described a method for estimating range and Doppler spread radar targets in amplitude domain at sub baud-length range-resolution using linear statistical inversion (Vierinen et al., 2008b). However, we did not use codes optimized for the targets that we analyzed. Also, we only briefly discussed code optimality. In this paper we will focus on optimal transmission codes for a target range resolution that is smaller than the minimum allowed baud-length. We will introduce a variant of phase coding called "fractional baudlength codes" that are useful for amplitude domain inversion

Correspondence to: J. Vierinen (juha.vierinen@iki.fi) of range and possibly Doppler spread targets, when a better resolution than the minimum allowed radar transmission envelope baud-length is required.

The method introduced in this study differs from the Frequency Domain Interferometry (FDI) (Kudeki and Stitt, 1987) method as it does not require the target scattering to originate from a very narrow layer within the radar scattering volume. Assuming that the target is indeed a narrow enough layer, the FDI method will probably perform better in terms of range resolution. However, it is feasible to combine fractional baud-length coding with FDI to obtain a shorter decoded pulse before the interferometry step.

In radar systems there is a limit to the smallest baud length, which arises from available bandwidth due to transmission system or licensing constraints. However, the transmission envelope can be timed with much higher precision than the minimum baud length. For example, the EISCAT UHF and VHF mainland systems in Troms $\varnothing$ are currently capable of timing the transmission envelope at $0.1 \mu$ s resolution, but the minimum allowed baud length is $1 \mu$ s. Thus, it is possible to use transmission codes with non-uniform baud-lengths that are timed with $0.1 \mu$ s accuracy, as long as the shortest baud is not smaller than $1 \mu \mathrm{s}$. This principle can then be used to achieve high resolution $(<1 \mu \mathrm{s})$ backscatter estimates with smaller variance than what would be obtained using a uniform baud-length radar transmission code with baud lengths that are integer multiples of $1 \mu \mathrm{s}$.

In this paper, we first derive the target backscatter amplitude estimation covariance for range and Doppler spread radar targets when estimating target parameters in amplitude domain. Then we define transmission code optimality for a given target. After this, we present two search strategies which can be used to find optimal transmission codes: an exhaustive search algorithm, and an optimization search algorithm. As an example, we study code optimality in the case of a simulated range spread coherent target. We also show an example of a real fractional baud-length coding measurement of a range spread meteor echo.

Published by Copernicus Publications on behalf of the European Geosciences Union. 


\section{Fractional baud-length code}

We will treat the problem in discrete time. The measurement sample rate is assumed to be the same as the required target range resolution.

A transmission envelope can be described as a baseband sequence of $L$ samples. If the transmission envelope has much less bauds $N_{\mathrm{b}} \ll L$ than samples, it is economical to represent the transmission code in terms of bauds. In this case, the envelope can be described in terms of the lengths $l_{k} \in \Gamma \subset \mathbb{N}$, phases $\phi_{k} \in \mathrm{P} \subset[0,2 \pi)$ and amplitudes $a_{k} \in \Lambda \subset[0,1] \subset \mathbb{R}$ of the bauds. We can define an arbitrary transmission envelope as

$\epsilon_{\mathrm{t}}=\sum_{j=1}^{N_{\mathrm{b}}}\left[t \in B_{j}\right] a_{j} e^{i \phi_{j}}$,

where [.] is the so called Iverson bracket, which evaluates to 1 if the logical expression is true - in this case when the index " $\mathrm{t}$ " is within the set of indices $B_{j}=\left\{1+\sum_{i=0}^{j-1} l_{i}, 2+\right.$ $\left.\sum_{i=0}^{j-1} l_{i}, \cdots, l_{j}+\sum_{i=0}^{j-1} l_{i}\right\}$ within baud $j$ and zero otherwise (additionally, we define $l_{0}=0$ ). In this study, the code power is always normalized to unity $\sum_{t=-\infty}^{\infty}\left|\epsilon_{t}\right|^{2}=1$, which means that the variances are comparable between transmission envelopes that deliver a similar amount of radar power. The codes can also be normalized otherwise, if comparison between two envelopes of different total power is needed.

The transmission waveform definition is intentionally as general as possible. Radar specific constraints can be imposed by defining the sets $\Gamma, \mathrm{P}$, and $\Lambda$. These will be discussed later on in Sect. 5.

\section{Target estimation variance}

The presentation here slightly differs from Vierinen et al. (2008b). Instead of a Fourier series, we will use B-splines to model the target backscatter.

Using discrete time and range, and assuming that our receiver impulse response is sufficiently close to a boxcar function that is matched to the sample rate, the direct theory for a signal measured from a radar receiver can be expressed as a sum of the range lagged transmission envelope multiplied by the target backscatter amplitude

$m_{t}=\sum_{r \in R} \epsilon_{\mathrm{t}-\mathrm{r}} \zeta_{r, t}+\xi_{t}$

Here $m_{t} \in \mathbb{C}$ is the measured baseband raw voltage signal, $R=\left\{R_{\min }, \ldots, R_{\max }\right\} \subset \mathbb{N}$ is the target range extent, $\epsilon_{\mathrm{t}} \in \mathbb{C}$ is the transmission modulation envelope, $\zeta_{r, t} \in \mathbb{C}$ is the range and time dependent target scattering coefficient and $\xi_{t} \in \mathbb{C}$ is measurement noise consisting of thermal noise and sky-noise from cosmic radio sources. The measurement noise is assumed to be a zero mean complex Gausian white noise with variance $\mathrm{E} \xi_{\mathrm{t}} \overline{\xi_{t^{\prime}}}=\delta_{t, t^{\prime}} \sigma^{2}$. Ranges $r$ are defined in round-trip

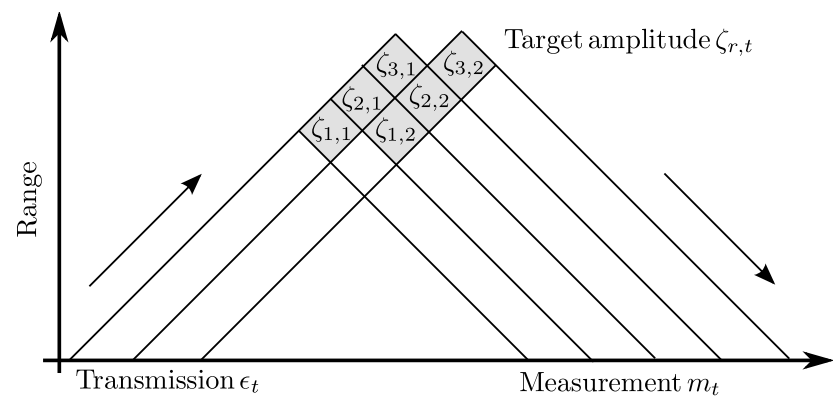

Fig. 1. Simplified range-time diagram of backscatter from a strong narrow region (notice that this is not in round-trip time). In this example there are two transmit samples and three ranges that cause backscatter. The gray area represents the area where the backscatter of one sample originates from, assuming boxcar impulse response. A longer impulse response will cause more range spreading.

time at one sample intervals, $t$ also denotes time in samples. By convention, we apply a range dependent constant $\frac{r}{2}$ delay, so that the range dependent backscatter amplitude is $\zeta_{r, t}$ instead of $\zeta_{r, t-\frac{r}{2}}$. Figure 1 depicts backscatter from three range gates probed with two transmission samples. To simplify matters, we use overlapping triangular range gates.

\subsection{Coherent target}

Now if the target backscatter is constant $\zeta_{r, t}=\zeta_{r}$, the measurement equation becomes a convolution equation

$m_{t}=\sum_{r \in R} \epsilon_{t-r} \zeta_{r}+\xi_{t}$,

which is the most common measurement equation for radar targets. Assuming that the target is sufficiently extended, this can be solved by filtering the measurements with a filter that corresponds to the frequency domain inverse of the transmission envelope (Sulzer, 1989; Ruprecht, 1989). However, for a finite range extent, the filtering approach is not always optimal as it does not properly take into account edge effects, such measurements missing due to ground clutter or receiver protection. A sufficiently narrow range extent also results in smaller estimation errors. In these cases, one should use a linear theory matrix that explicitely defines the finite range extent. We will define this as a special case of the incoherent backscatter theory presented next.

\subsection{Incoherent target}

If the target backscatter is not constant, the range dependent backscatter $\zeta_{r, t}$ has to be modeled in some way in order to make the estimation problem solvable. One natural choice is to assume that the target backscatter is a band-limited signal, which can be modeled using a B-spline (de Boor, 1978). Our model parameters will consist of $N_{\mathrm{s}}$ control points that model the backscatter at each range of interest. The frequency domain characteristics are defined by the spacing of 
the knots and the order of the spline $n$. Using the definition of B-splines, the target backscatter $\zeta_{r, t}$ is modeled using the parameters $P_{r, k} \in \mathbb{C}$ as:

$\hat{\zeta}_{r, t}=\sum_{k=0}^{N_{\mathrm{s}}-1} P_{r, k} b_{k, n}\left(\frac{t-1}{L-1}\right)$,

where $b_{k, n}(\cdot)$ is the $\mathrm{B}$-spline basis function and coefficients $P_{r, k}$ are the control points with $k \in\left\{1, \ldots, N_{\mathrm{s}}\right\}$. We assume that the control points are evenly spaced and that the endpoints contain multiple knots in order to ensure that the second order derivatives are zero at both ends of $\hat{\zeta}_{r, t}$. We also define a special case of one spline control point as $\hat{\zeta}_{r, t}=P_{r}=$ $\zeta_{r}$. This corresponds to a completely coherent target.

When Eq. (4) is substituted into Eq. (2), we get

$m_{t}=\sum_{r \in R} \sum_{k=0}^{N_{s}-1} P_{r, k} \epsilon_{t-r} b_{k, n}\left(\frac{t-1}{L-1}\right)+\xi_{t}$.

This model is linear in respect to the parameters $P_{r, k}$ and one can conveniently represent it in matrix form as

$\boldsymbol{m}=\mathbf{A} \boldsymbol{x}+\boldsymbol{\xi}$,

where $\boldsymbol{m}=\left[m_{1}, \ldots, m_{N}\right]^{\mathrm{T}}$ is the measurement vector, $\mathbf{A}$ is the theory matrix containing the $\epsilon_{t-r} b_{k, n}(\cdot)$ terms, $\boldsymbol{x}=$ $\left[P_{1,1}, P_{1,2}, \ldots, P_{N_{r}, N_{\mathrm{s}}}\right]^{\mathrm{T}}$ is the parameter vector containing the control points and $\xi=\left[\xi_{1}, \ldots, \xi_{N}\right]^{\mathrm{T}}$ is the error vector with the second moment defined as

$\mathrm{E} \xi \xi^{\mathrm{H}}=\boldsymbol{\Sigma}=\operatorname{diag}\left(\sigma^{2}, \ldots, \sigma^{2}\right)$.

The number of parameters is the number of ranges $N_{\mathrm{r}}$ times the number of B-spline control points $N_{\mathrm{s}}$ per range. The number of measurements $N=N_{\mathrm{r}}+L-1$ is a sum of target ranges and transmission envelope length $L$. As long as $N \geq$ $N_{\mathrm{r}} N_{\mathrm{s}}$ and the theory matrix has sufficient rank, the problem can be solved using statistical linear inversion. In practice, the number of model parameters that can be succesfully modeled with sufficiently small error bars depends on the signal to noise ratio. The estimation of strong range and Doppler spread echos is shown in Vierinen et al. (2008b). Figure 2 shows an example theory matrix for a target range extent $N_{\mathrm{r}}=14$ with $N_{\mathrm{S}}=8$ spline guide points per range. The transmission code is a uniform baud-length 13-bit Barker code with baud length $l_{j}=10$.

The probability density for Eq. (6) can be written as:

$p(\boldsymbol{m} \mid \boldsymbol{x}) \propto \exp \left(-\frac{1}{\sigma^{2}}\|\boldsymbol{m}-\mathbf{A} \boldsymbol{x}\|^{2}\right)$

and assuming constant valued priors, the maximum a posteriori (MAP) estimate, i.e., the peak of $p(\boldsymbol{m} \mid \boldsymbol{x})$ is

$\boldsymbol{x}_{\mathrm{MAP}}=\left(\mathbf{A}^{\mathrm{H}} \mathbf{A}\right)^{-1} \mathbf{A}^{\mathrm{H}} \boldsymbol{m}$

and the a posteriori covariance is:

$\boldsymbol{\Sigma}_{\boldsymbol{p}}=\sigma^{2}\left(\mathbf{A}^{\mathrm{H}} \mathbf{A}\right)^{-1}$.

\section{B-Spline theory matrix}

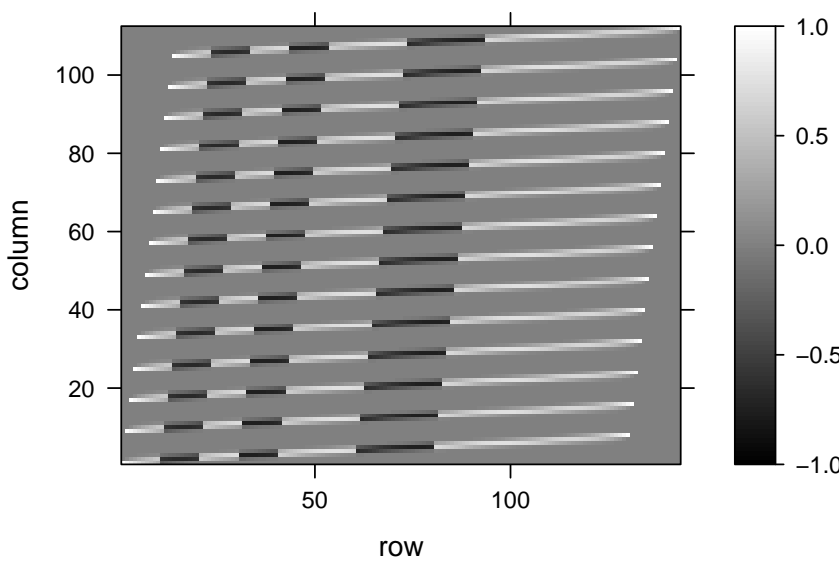

Fig. 2. A theory matrix for a range and Doppler spread target with $N_{\mathrm{r}}=14$ range gates and $N_{\mathrm{S}}=8 \mathrm{~B}$-spline guide points per range. The code is a simple 13-bit Barker code with 10 samples per baud.

\subsection{Infinitely extended coherent target}

In the special case of an infinitely extended coherent target $\left(N_{\mathrm{s}}=1\right)$, the matrix A becomes a convolution operator and the problem can be efficiently solved in frequency domain and numerically evaluated using FFT (Cooley and Tukey, 1965). This case has been extensively discussed by, e.g., Lehtinen et al. (2008); Vierinen et al. (2006); Lehtinen et al. (2004) and Ruprecht (1989). The covariance matrix will be an infinitely extended Toeplitz matrix with rows ${ }^{1}$ :

$\Sigma_{t}=\lim _{M \rightarrow \infty} \frac{1}{M} \mathcal{F}_{M}^{-1}\left\{\left(\mathcal{F}_{M}\left\{\sum_{\tau=-\infty}^{\infty} \epsilon_{\tau} \overline{\epsilon_{\tau-t}}\right\}\right)^{-1}\right\}$.

This result is also a fairly good approximation for a sufficiently long finite range extent, differing only near the edges. However, this result is not valid for a sufficiently narrow finite range extent or when the target also has Doppler spread. Also, it is not even possible to calculate the covariance matrix in this way for uniform baud-length codes when the baudlength is larger than the target resolution. The reason for this is that for an infinitely extended target there will be zeros in the frequency domain representation of the transmission envelope and because of this, the covariance matrix is singular. Even in the case of a finite range extent, all codes with uniform baud-length result in a theory matrix with strong linearly dependent components. An example of this is shown in Sect. 6. When using non-uniform baud-lengths the problem can be avoided, since in this way it is possible to form a code

\footnotetext{
${ }^{1}$ The index $t$ refers to the column of the matrix row. Operators $\mathcal{F}_{M}$ and $\mathcal{F}_{M}^{-1}$ are the forward and reverse discrete Fourier transforms of length $M$. In practice the covariance can be approximated numerically with sufficiently large values of $M$.
} 
without zeros in the frequency domain. This is somehwhat similar to random alias-free sampling (Shapiro and Silverman, 1960). Another analogy can be found in the use of aperiodic radar interpulse periods to overcome range-Doppler ambiguities, e.g., (Farley, 1972; Uppala and Sahr, 1994; Pirttilä and Lehtinen, 1999).

\section{Code optimality}

The performance of a certain code is determined by the target parameter estimation errors. These on the other hand are determined by the a posteriori covariance matrix in Eq. (10). Because we assume uniform priors, the covariance matrix is fully determined by the target model (i.e., assumed target characteristics) in theory matrix $\mathbf{A}$. The theory matrix $\mathbf{A}$ contains the transmission envelope and therefore it affects the covariance matrix. The task of code optimization is to find a covariance matrix that produces the best possible estimates of the target.

In terms of the theory of comparison of measurements (Piiroinen, 2005), a code $\epsilon_{1}$ is in every situation better than some other code $\epsilon_{2}$ only if the difference of their corresponding covariance matrices $\boldsymbol{\Sigma}_{\mathbf{2}}-\boldsymbol{\Sigma}_{\mathbf{1}}$ is positive definite. Even though it might be feasible use this as a criterion in a code search, we chose a more pragmatic approach where we construct a function that maps the the covariance matrix to a real number $\Omega: \mathbb{R}^{N_{p} \times N_{p}} \rightarrow \mathbb{R}$ while still retaining some of the information contained in the covariance matrix. One such map is the trace of the covariance matrix $\Omega(\boldsymbol{\Sigma})=\operatorname{tr}(\boldsymbol{\Sigma})$, which is called A-optimality in terms of optimal statistical experiment design. This has the effect of minimizing the average variance of the model parameters. We will use this criterion throughout this paper. Refer to, e.g., Pukelsheim (1993) for more discussion on optimization criteria.

For infinitely extended fully coherent targets, the trace of the covariance matrix is infinite, but one can use the diagonal value of one row of the covariance matrix. Because it is of Toeplitz form, all diagonal values are the same, and this will correspond to A-optimality.

\section{Code search}

The transmission envelope consisting of $N_{\mathrm{b}}$ bauds is fully described by the baud lengths $l_{k} \in \Gamma \subset \mathbb{N}$, phases $\phi_{k} \in \mathrm{P} \subset$ $[0,2 \pi)$ and amplitudes $a_{k} \in \Lambda \subset[0,1] \subset \mathbb{R}$. These form the set of parameters to optimize

$\left(l_{k}, \phi_{k}, a_{k}\right) \subset \Gamma^{N_{\mathrm{b}}} \times \mathrm{P}^{N_{\mathrm{b}}} \times \Lambda^{N_{\mathrm{b}}}$.

In addition to this, the number of bauds $N_{\mathrm{b}}$ in a code of length $L$ need not be fixed, as this depends on the lengths of the individual bauds $l_{k}$.

For reasonably short codes with sufficiently small number of phases it might be possible to perform an exhaustive

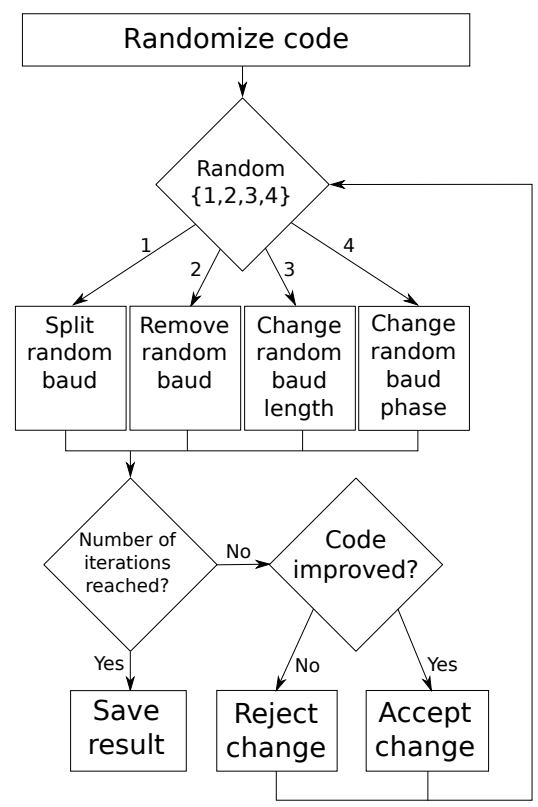

Fig. 3. Simplified block diagram of the optimization algorithm.

search. This consists of first determining all the different ways to divide a code of length $L$ into bauds of lengths $l_{k}$. After this, all unique orderings of the baud-lengths and permutations of phases and amplitudes need to be traversed. The problem of determining the different combinations of baud lengths amounts to the problem of generating all integer partitions for $L$ (Kelleher and O'Sullivan, 2009). When additional constraints to baud lengths are applied, the problem is called the multiply restricted integer partitioning problem (Riha and James, 1976). An efficient algorithm for iterating through restricted partitions has been described by Riha and James (1976).

The exhaustive approach fails already for reasonably small problems due to the catastrophic growth of the search space. Therefore we have to resort to some optimization method in order to find optimal codes. Optimization methods have been previously used for code searches at least by Sahr and Grannan (1993), and Nikoukar (2010). Our approach for finding optimal codes is based on the simulated annealing method (Kirkpatrick et al., 1983).

The optimization procedure that we have developed can be used to find well performing non-uniform baud-length codes, given a set of constraints. The constraints are given as the set of allowed baud lengths $\Gamma$, the set of allowed phases $P$ and the set of allowed amplitudes $\Lambda$. We have previously used a similar algorithm to optimize codes for infinitely extented coherent targets and lag-profile inversion of incoherent scatter radar (Vierinen et al., 2006, 2008a).

The main principle of the algorithm is very simple. We first randomize a code $E_{0} \equiv\left(l_{k}, \phi_{k}, a_{k}, N_{\mathrm{b}}\right)$ that meets the given constraints. Next, for a certain number of iterations we incrementally attempt to improve this code with small 
random changes $E^{\prime}=\delta E_{i}$. Here $\delta$ is an operator that slightly modifies $E$ in some way, while conforming to the constraints imposed on the code. If any of these changes results in a code that is better, we then save these parameters $E_{i+1}=E^{\prime}$ and continue to the next iteration. In order to reduce the chance of the algorithm from getting stuck in a local minima, we also sometimes (by a small random chance) allow changes that do not improve the code. In order to achieve convergence, the magnitude of the random changes $\delta E$ is decreased as the iteration advances. The algorithm is depicted in Fig. 3. The small incremental changes that we use are:

1. Split random baud. Select a long enough baud in the code and split it into two bauds. Retain the original amplitude and phase on one of these bauds and slightly randomly modify them on the other baud.

2. Remove a random baud. Increase a randomly selected baud length.

3. Change the length of a baud. Increase the length of one randomly selected baud and decrease the length of another randomly selected baud to maintain code length.

4. Change random baud phase and amplitude. Select an arbitrary baud and slightly change phase and amplitude.

These incremental changes are designed in a way that they always conform to the criteria imposed on the transmission code. If this is not possible (i.e., if it is not possible to add a new baud), the code remains unchanged. The implementation of "slight change" depends on the constrains placed on the code. E.g., in the case of binary phase codes with constant amplitude, a $180^{\circ}$ phase flip would correspond to a slight phase change, while the amplitudes would remain unchanged. For a polyphase code, the slight change in phase could be a small random change in the original phase $\phi_{k}^{\prime}=\phi_{k}+\epsilon_{\phi}$, where $\epsilon_{\phi}$ would be a small random number.

In order to initially randomize a code, we start with any phase code that conforms with the constraints of baud lengths, phases and amplitudes. This can be hard coded. We then perform a certain number of the same incremental changes that we use in the optimization procedure, except that we accept all of the changes.

\section{Example: range spread coherent target}

To demonstrate the performance of non-uniform baud-length codes when estimating a target at sub-baud resolution, we simulated an echo using a constant amplitude binary phase non-uniform baud-length code and traditional uniform baudlength constant amplitude binary phase code of the same length. In this example, we analyze a 10 sample wide target at the resolution of one sample. The non-uniform baud-length code was an optimized 11-bit code with baud lengths $\{12,12,12,12,10,13,10,11,11,15,12\}$ and phases
$\{1,-1,1,-1,1,-1,1,-1,1,-1,-1\}$. The smallest allowed baud-length was 10 samples. For comparison we used the well known 13-bit Barker code with a baud-length of 10 samples. Both simulations had the same instance of measurement noise SNR $=3$ and the same target amplitudes, which in this case was an instance of the complex Gaussian random $\mathcal{N}_{\mathbb{C}}(0,1)$ process.

The results are shown in Fig. 4. It is evident that the nonuniform baud-length code performs better in terms of estimation errors. It is also evident that the Barker code suffers from the fact that every baud is the same length - if the range extent would have been infinite, the covariance matrix would have been singular. Now the covariance matrix is only near-singular. This is seen as large off-diagonal stripes in the 13-bit Barker code covariance matrix and correlated errors. In the case of the 11-bit fractional code the off-diagonal elements are more uniform and the variance is also smaller.

\section{Example: meteor echo structure}

During the 15-19 November 2009 Leonid meteor campaign, we used a set of 53 optimized fractional baud-length codes with $0.5 \mu$ s fractional resolution and $5 \mu$ s minimum baud length. The transmission pulse length was $371 \mu$ s. The data was sampled at sampled at $2 \mathrm{MHz}$ sample rate. The large number of pulses, together with the fairly long baud-length allowed simultaneous analysis of space debris and the ionosphere, while not sacrificing too much in terms of meteor head echo parameter estimation accuracy. We used the EISCAT UHF radar located in Tromso, with the $32 \mathrm{~m}$ antenna beam pointed approximately $99 \mathrm{~km}$ above Peera, Finland, giving a zenith angle of about $42^{\circ}$. The radar peak power was approximately $1.4 \mathrm{MW}$.

During this campaign, one of the observed "strange" meteor echos was an echo at approximately $60 \mathrm{~km}$. The meteor head (or the dense cloud of plasma) is first seen decelerating from about $1 \mathrm{~km} \mathrm{~s}^{-1}$ to $0 \mathrm{~km} \mathrm{~s}^{-1}$. After this, several disjoint trail-like structures persist for nearly $2 \mathrm{~s}$.

The meteor head echo was detected by searching for the maximum likelihood parameters for a single echo moving point-target model

$m_{t}=\sigma \epsilon_{t-R_{0}} \exp \{i \omega t\}+\xi_{t}$,

where $\sigma$ is the backscatter amplitude, $\omega$ is the Doppler shift and $R_{0}$ is range ( $\xi_{t}$ denotes receiver noise). The maximum likelihood parameters were obtained using a grid search of the likelihood function resulting from the measurement model. This is necessary as the Doppler shift is usually significant for meteor head echos at $929 \mathrm{MHz}$ with such a long pulse.

The detected echo was then analyzed using a coherent spread target model (Eq. 3), which assumes that the backscatter comes from an extended region with a uniform Doppler 

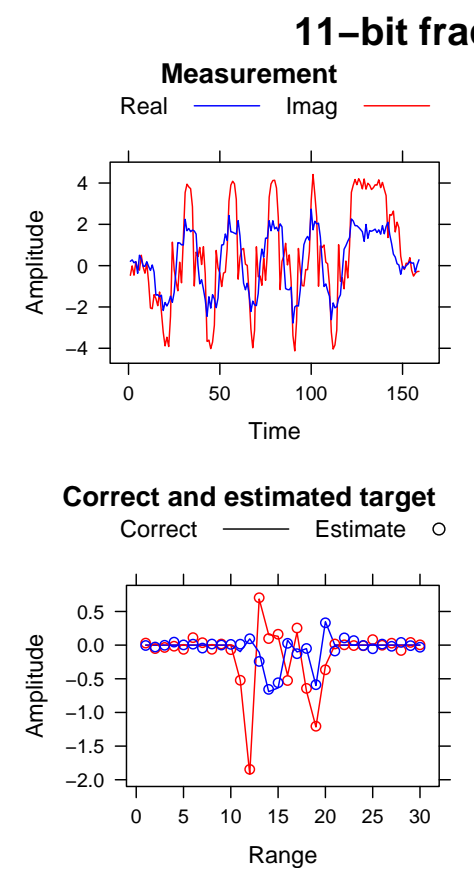

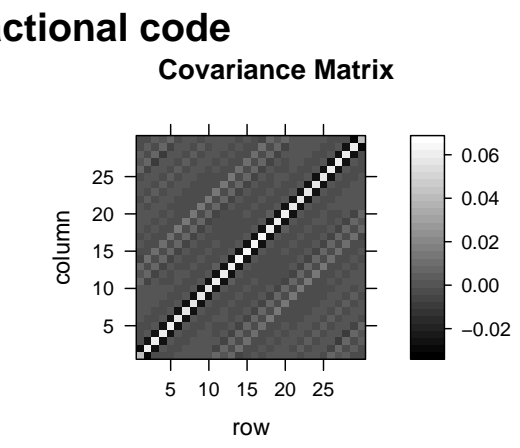

Error

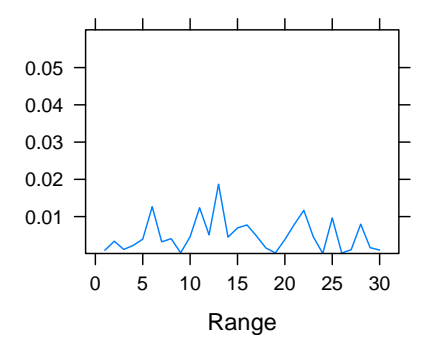

13-bit Barker code

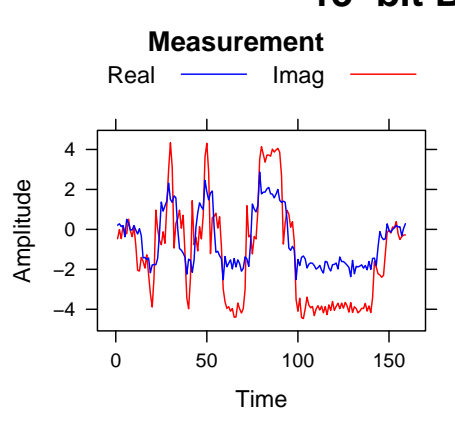

Correct and estimated target Correct - Estimate 0

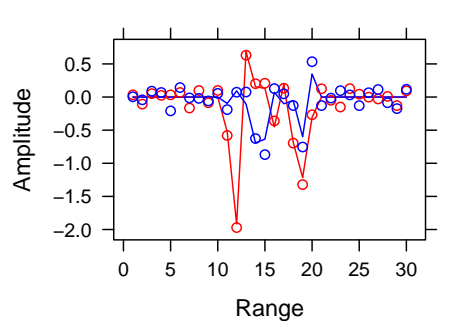

Covariance Matrix

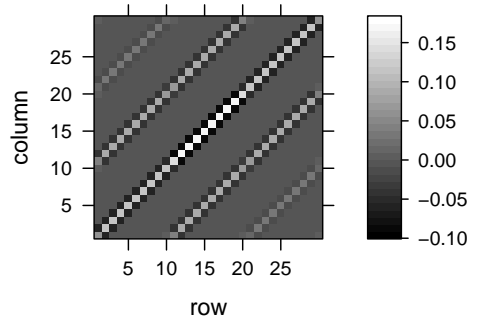

Error

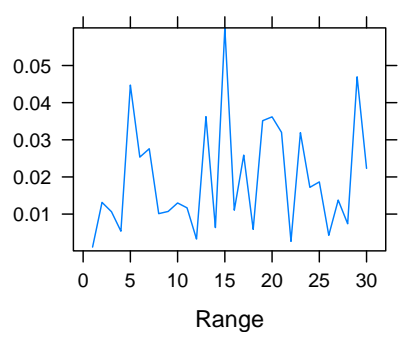

Fig. 4. Simulated coherent echo from a 11-bit non-uniform baud-length code that is $13 \mu$ s long and the smallest baud-length is $1 \mu$ s. When compared to the performance of a uniform baud-length 13-bit Barker code with $1 \mu$ s baud length, the performance is again better. The simulated target length is $10 \mu$ s and $\mathrm{SNR}=3$.

shift. The analysis resulted in the generalized linear leastsquares parameter estimate for range dependent complex backscatter amplitude, which in other words is a range sidelobe-free estimate of the target backscatter. The range resolution was $0.5 \mu \mathrm{s}$, even though the minimum baud-length of the code was $5 \mu$ s. The Doppler shift obtained from the point-target estimate was used in the spread-target estimate - although this correction was not significant after the first $0.2 \mathrm{~s}$ of the echo as the Doppler shift was very close to 0 .

The results in of the moving point and spread target estimates are shown in Fig. 5. The moving point-model indicates that after the initial deceleration, the trails have nearly zero 
Doppler shift. The spread target results show that there are up to seven different layers separated in altitude. The strongest layer also shows range spread up to $500 \mathrm{~m}$. Had a uniform baud length code with $5 \mu$ s bauds been used, the a posteriori variance would have been approximately twice larger.

This is the first time that such echos have been seen in EISCAT UHF observations. As micrometeoroids do not reach such a low altitude, one possible explanation is that this is a larger object. Perhaps a bolide. Because the altitude of this mono-static detection was obtained assuming that the target was within the main lobe of the antenna, another possible explantion is that this is a far side lobe detection of a combined meteor head and specular trail echo directly above the radar at approximately $85-90 \mathrm{~km}$ altitude. However, this would require the target to be approximately $45^{\circ}$ off axis.

Meteor trail echos are not typically observed in EISCAT UHF observations as the high latitude location does not allow observing magnetic field-aligned irregularities. Also, the trail electron density is typically too small to be observed at UHF frequencies, making observation of specular trail echos unlikely.

Recent observations at Jicamarca (Malhotra and Mathews, 2009) have indicated a new type of scattering mechanism that does not yet have a physical explanation. These so called Low Altitude Trail Echos (LATE) seem to have no preference to the angle between the magnetic field and radar beam. They also have different characteristics than specular trail echos as they are typically observed only at low altitudes, usually together with head echos. Malhotra and Mathews (2009) suggest that these echos are produced as a by-product of fragmentation. Our results show that there are at least seven distinct layers, which is an indication that the meteor has fragmented multiple times. However, this event is different from those described by Malhotra and Mathews in the sense that this trail is at a much lower altitude $(60-65 \mathrm{~km})$ and also the trail is more long lasting. So it is difficult to say if the same scattering mechanism applies here.

\section{Conclusions}

In this paper, we first describe the statistical theory of estimating coherent and incoherent radar targets in amplitude domain. We then study target amplitude domain estimation variance for different codes. Using these results, we show that when sub baud-length resolution is needed, a transmission code that has non-uniform baud length results smaller estimation variance than a traditional code with uniform baud lengths. We then discuss a numerical method for finding suitable constrained transmission codes. The principles are demonstrated using simulated and real coherent radar echos.

The main application of non-uniform baud-length coding will be in cases where there is good SNR and sufficient receiver bandwidth, but a limited transmission bandwidth. Although the examples in this study only deal with coher-
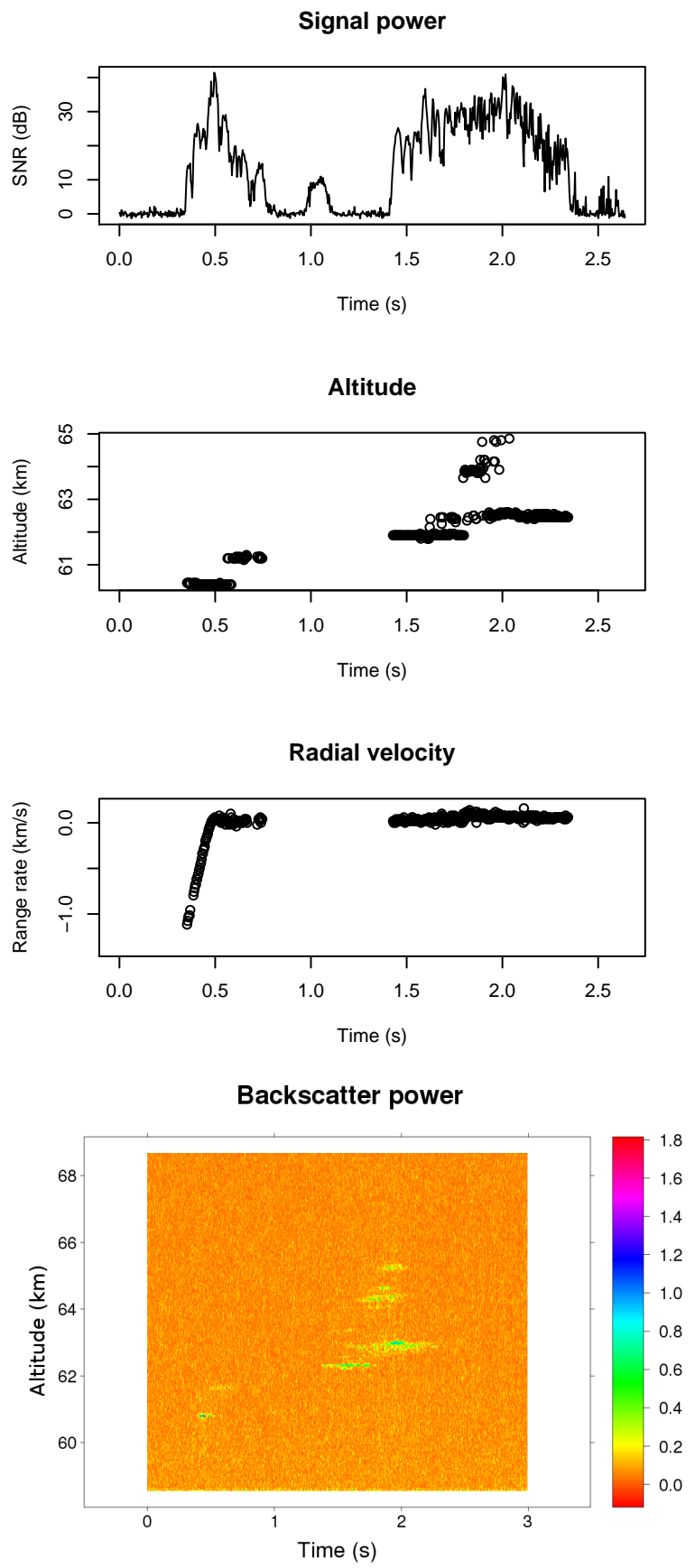

Fig. 5. A low-altitude meteor echo at $60 \mathrm{~km}$ seen with the EISCAT UHF radar on 19 November 2009 at 05:16 UT during a lowelevation meteor experiment. The meteor echo can been decelerating from $1 \mathrm{~km} \mathrm{~s}^{-1}$ down to 0 , and then then echos from a trail-like structure are seen for a while. The three top panels show the results from a moving point-target model that determines the most likely range, Doppler shift and power of a point target. The fourth panel shows the range resolved backscatter power from a spread target model. After the initial head echo, many layers appear at altitudes above the initial detection. Most of the layers show 100-400 m range spread. 
ent targets, the considerations also apply for amplitude domain estimation of strong range and Doppler spread (incoherent) echos, such as the ones described in Vierinen et al. (2008b). Examples of practical use cases include Lunar measurements, range spread meteor trail studies, and artificial ionospheric heating induced enhanced ion- and plasma-line echos.

Non-uniform baud-lengths are also advantageous for multi-purpose high power large aperture radar experiments where one mainly observes targets that benefit from longer baud lengths (e.g., ionospheric plasma or space debris), but where one would still want to be able to analyze strong targets at sub-baud resolution.

Although we have only studied the non-uniform baudlength coded transmission envelope performance in the case of amplitude domain target estimation, the same principles can also be applied to find optimal high resolution transmission codes for lag-profile inversion (Virtanen et al., 2008c) using estimation variance calculations that can be found e.g., in Lehtinen et al. (2008).

Acknowledgements. Editor-in-Chief M. Pinnock thanks J. Sahr and another anonymous referee for their help in evaluating this paper.

\section{References}

Cooley, J. W. and Tukey, J. W.: An algorithm for the machine calculation of complex fourier series, Math. Comput., 19, 297-301, 1965.

de Boor, C.: A Practical Guide to Splines, 114-115 pp., SpringerVerlag, 1978.

Farley, D. T.: Multiple-pulse incoherent-scatter correlation function measurements, Radio Sci., 7(6), 661-666, 1972.

Kelleher, J. and O'Sullivan, B.: Generating all partitions: A comparison of two encodings, CoRR, abs/0909.2331, 2009.

Kirkpatrick, S., Gelatt, C. D., and Vecchi, M. P.: Optimization by Simulated Annealing, Science, 220(4598), 671-680, doi:10.1126/science.220.4598.671, 1983.

Kudeki, E. and Stitt, G. R.: Frequency domain interferometry: A high resolution radar technique for studies of atmospheric turbulence, Geophys. Res. Lett., 14(3), 198-201, 1987.

Lehtinen, M. S., Damtie, B., and Nygrén, T.: Optimal binary phase codes and sidelobe-free decoding filters with application to incoherent scatter radar, Ann. Geophys., 22, 1623-1632, doi:10.5194/angeo-22-1623-2004, 2004.

Lehtinen, M. S., Virtanen, I. I., and Vierinen, J.: Fast comparison of IS radar code sequences for lag profile inversion, Ann. Geophys., 26, 2291-2301, doi:10.5194/angeo-26-2291-2008, 2008.
Malhotra, A., and Mathews, J. D.: Low-altitude meteor trail echoes, Geophys. Res. Lett., 36, L21106, doi:10.1029/2009GL040558, 2009.

Nikoukar, R.: Near-optimal inversion of incoherent scatter radar measurements: coding schemes, processing techniques, and experiments, $\mathrm{PhD}$ thesis, University of Illinois at UrbanaChampaign, 2010.

Piiroinen, P.: Statistical measurements, experiments and applications, Academia Scientiarum Fennica, 2005.

Pirttilä, J. and Lehtinen, M.: Solving the range-doppler dilemma with ambiguity-free measurements developed for incoherent scatter radars, COST 75, Advanced Weather radar systems, International seminar, pp. 557-568, 1999.

Pukelsheim, F.: Optimal Design of Experiments, John Wiley \& Sons, 1993.

Riha, W. and James, K. R.: Algorithm 29 efficient algorithms for doubly and multiply restricted partitions, Computing, 16, 163168, doi:10.1007/BF02241987, 1976.

Ruprecht, J.: Maximum-Likelihood Estimation of Multipath Channels, PhD thesis, Swiss federal institute of technology, 1989.

Sahr, J. D. and Grannan, E. R.: Simulated annealing searches for long binary phase codes with application to radar remote sensing, Radio Sci., 28(6), 1053-1055, doi:10.1029/93RS01606, 1993.

Shapiro, H. S. and Silverman, R. A.: Alias-free sampling of random noise, Journal of the Society for Industrial and Applied Mathematics, 8(2), 225-248, 1960.

Sulzer, M. P.: Recent incoherent scatter techniques, Adv. Space Res., 9, 153-162, 1989.

Uppala, S. V. and Sahr, J. D.: Spectrum estimation of moderately overspread radar targets using aperiodic transmitter coding, Radio Sci., 29, 611-623, 1994.

Vierinen, J., Lehtinen, M. S., Orispää, M., and Damtie, B.: General radar transmission codes that minimize measurement error of a static target, arxiv:physics/0612040v1, published on-line at arxiv.org, 2006.

Vierinen, J., Lehtinen, M. S., Orispää, M., and Virtanen, I. I.: Transmission code optimization method for incoherent scatter radar, Ann. Geophys., 26, 2923-2927, doi:10.5194/angeo-262923-2008, 2008a.

Vierinen, J., Lehtinen, M. S., and Virtanen, I. I.: Amplitude domain analysis of strong range and Doppler spread radar echos, Ann. Geophys., 26, 2419-2426, doi:10.5194/angeo-26-24192008, 2008b.

Virtanen, I. I., Lehtinen, M. S., Nygrén, T., Orispää, M., and Vierinen, J.: Lag profile inversion method for EISCAT data analysis, Ann. Geophys., 26, 571-581, doi:10.5194/angeo-26-571-2008, 2008c. 